\title{
Steady State Analysis of Class-E Amplifier with non-linear capacitor by means of discrete-time techniques.
}

\author{
Francisco del Aguila López, Pere Pàlà Schönwälder, Jordi Boñet Dalmau and Rosa Giralt Mas \\ DEPARTAMENT DE TEORIA DEL SENYAL I COMUNICACIONS \\ UNIVERSITAT POLITĖCNICA DE CATALUNYA \\ Av. Bases de Manresa, 61-73, Manresa 08240, Spain \\ aguila@tsc.upc.es
}

\section{ABSTRACT}

A, new method to determine the steady state response of switched nonlinear circuits is proposed: The method is based on a Gear discretization of the circuit equations. Additional samples of the waveform are used to describe the circuit when switching from one topology to another. Results are presented for a class $\mathrm{E}$ resonant inverter.

\section{INTRODUUCTION}

During the last years switched circuits have become widely used due to its efficiency in power supply circuits and RF amplifiers. Analysis tools are very important for the designer because they allow predicting the behaviour of circuits accurately. In many applications the knowledge of transient behaviour is secondary, the main interest being in the steady state waveforms. In this paper a new method for the steady state analysis of switched circuits with non-linear elements is presented. The technique is based on the time-domain discretization of the resulting nonlinear circuit equations. The method is applied to the class-E amplifier.

\section{METHOD DESCRIPTION}

A paradigm of the class of circuits to analyse consists of an ideal switch and a non-linear element connected by a linear network. The class- $\mathrm{E}$ amplifier depicted in fig. 1 is such a circuit. The ideal switch is modeled as a short-circuit in $\mathrm{ON}$ state and an open-circuit in OFF state. The switching instant is known and externally controlled. We can divide the system in two topologies depending " on 'the 'switch's state. In each topology, the control variable $v$ may be obtained solving

$$
\begin{aligned}
& V(s)=H_{O N}(s) Q_{D}(V)+H_{2 O N}(s) V_{D D}(s) \\
& V(s)=H_{1 O F F}(s) Q_{D}(V)+H_{2 O F F}(s) V_{D D}(s)
\end{aligned}
$$

Once $\mathrm{y}$ is known, any other circuit variable is easily determined.

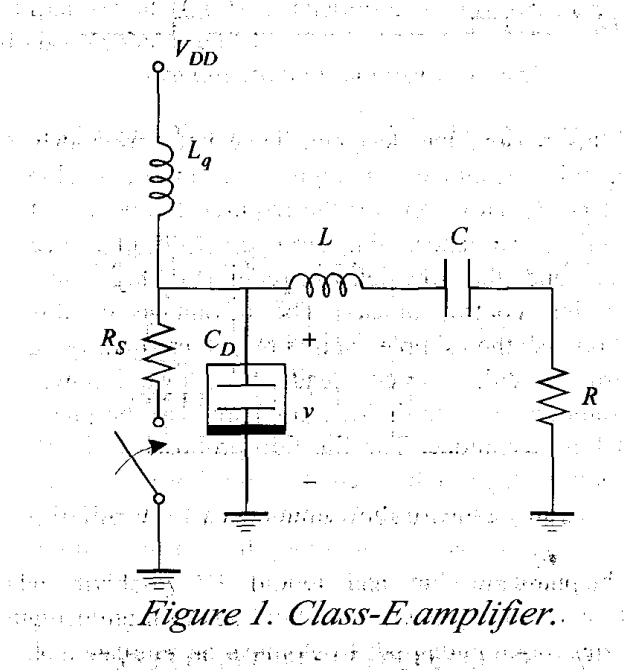

Non-linear inductors or capacitors are best described by their behaviour in terms of flux or charge variables. Function $Q_{D}(V)$ is the charge of the capacitor $C_{D}$ and $H_{k}$ are rational functions. As a consequence we can rewrite the equations as

$$
\begin{aligned}
& D_{O N}(s) V(s)+N_{1 O N}(s) Q_{D}(V)+N_{2 O N}(s) V_{D D}(s)=0 \\
& D_{\text {OFF }} K(s)+N_{1 O F F}(s) Q_{D}(V)+N_{2 O F F}(s) V_{D D}(s)=0
\end{aligned}
$$

Where $D(s)$ and $N_{k}(s)$ are polynomials. The control variable of the non-linearity $V(s)$ is the only unknown in these equations.

An extension of the method in [1] is proposed to solve this equation. First, the derivatives of $v(t)$ and $q_{D}(v)$ are approximated using gear dicretizations. For 
Gear-1 discretization $\quad \dot{v}_{n}=\frac{v_{n}-v_{n-1}}{\Delta}$ and for Gear-2 $\dot{v}_{n}=\frac{3 v_{n}-4 v_{n-1}+v_{n-2}}{2 \Delta}$.

As a result, equation (2) may be rewriter in the form

$\sum_{0}^{m} a_{n-i} v_{n-i}+\sum_{0}^{m} b_{n-i} q_{D}\left(v_{n-i}\right)+c=0$

Where the only unknowns are the samples of $v(t)$.

Numeric resolution of (3) allows computing the waveform in each state, once the initial conditions are determined.

When changing from one topology to the other, the state variables $\left[v_{C_{D}}, v_{C}, i_{L}, i_{L_{q}}\right]$ are continuous. Next, we derive a relation between $\left[v_{C_{D}}, v_{C}, i_{L}, i_{L_{q}}\right\rfloor$ and $\left(v_{n}, q_{D}\left(v_{n}\right) \ldots\right)$ that allows expressing state variable continuity in terms of $\left(v_{n}, q_{D}\left(v_{n}\right) \ldots\right)$.

Consider the equations describing the circuit behaviour for the $\mathrm{ON}$ state:

$\left[\begin{array}{l}\dot{q}_{D} \\ \dot{\nu}_{C} \\ \dot{i}_{L} \\ \dot{i}_{L_{q}}\end{array}\right]=\left[\begin{array}{cccc}\frac{-1}{R_{S}} & 0 & -1 & 1 \\ 0 & 0 & \frac{1}{C} & 0 \\ \frac{1}{L} & \frac{-1}{L} & \frac{-R}{L} & 0 \\ \frac{-1}{L_{V}} & 0 & 0 & 0\end{array}\right]\left[\begin{array}{c}v_{C_{D}} \\ v_{C} \\ i_{L} \\ i_{L_{q}}\end{array}\right]+\left[\begin{array}{c}0 \\ 0 \\ 0 \\ \frac{V_{C C}}{L_{q}}\end{array}\right]$

Repeatedly deriving (4) the relations expressed in (5) (see below) may be obtained.

The next step consists in computing the discretization matrix $\mathbf{D}$ relating $v, \dot{v}, \ldots$ to the samples of $v$ and $q_{D}, \dot{q}_{D}, \ldots$ to the samples of $q_{D}$. For the Gear-1 discretization scheme, the discretization matrix $\mathbf{D}=\mathbf{D}_{\mathbf{1}}$ is obtained from: $\left[\begin{array}{c}v(t) \\ \dot{v}(t) \\ \ddot{v}(t) \\ \dddot{v}(t)\end{array}\right]=\left[\begin{array}{cccc}0 & 0 & 0 & 1 \\ 0 & 0 & \frac{-1}{\Delta} & \frac{1}{\Delta} \\ 0 & \frac{1}{\Delta^{2}} & \frac{-2}{\Delta^{2}} & \frac{1}{\Delta^{2}} \\ \frac{-1}{\Delta^{3}} & \frac{3}{\Delta^{3}} & \frac{-3}{\Delta^{3}} & \frac{1}{\Delta^{3}}\end{array}\right]\left[\begin{array}{c}v_{n-3} \\ v_{n-2} \\ v_{n-1} \\ v_{n}\end{array}\right]$

For higher order discretization schemes there are several degrees of freedom when computing $\nu$ from $\hat{v}$. In this case, additional restrictions may be set. One approach is to consider that the behaviour of the derivatives at the current and the previous sample is the same (7). This approach yields a matrix $\mathbf{D}=\mathbf{D}_{\mathbf{2 2}}$ as:

$\left[\begin{array}{c}x(t) \\ \dot{v}(t) \\ \ddot{v}(t) \\ \dddot{v}(t) \\ v^{+}(t) \\ \dot{v}^{+}(t) \\ \ddot{v}^{+}(t) \\ \dddot{v}^{+}(t)\end{array}\right]=\left[\begin{array}{cccccccc}0 & 0 & 0 & 0 & 0 & 0 & 1 & 0 \\ 0 & 0 & 0 & 0 & \frac{1}{2 \Delta} & \frac{-4}{2 \Delta} & \frac{3}{2 \Delta} & 0 \\ 0 & 0 & \frac{1}{4 \Delta^{2}} & \frac{-8}{4 \Delta^{2}} & \frac{22}{4 \Delta^{2}} & \frac{-24}{4 \Delta^{2}} & \frac{9}{4 \Delta^{2}} & 0 \\ \frac{1}{8 \Delta^{3}} & \frac{-12}{8 \Delta^{3}} & \frac{57}{8 \Delta^{3}} & \frac{-136}{8 \Delta^{3}} & \frac{171}{8 \Delta^{3}} & \frac{-108}{8 \Delta^{3}} & \frac{27}{8 \Delta^{3}} & 0 \\ 0 & 0 & 0 & 0 & 0 & 0 & 0 & 1 \\ 0 & 0 & 0 & 0 & 0 & \frac{1}{2 \Delta} & \frac{-4}{2 \Delta} & \frac{3}{2 \Delta} \\ 0 & 0 & 0 & \frac{1}{4 \Delta^{2}} & \frac{-8}{4 \Delta^{2}} & \frac{22}{4 \Delta^{2}} & \frac{-24}{4 \Delta^{2}} & \frac{9}{4 \Delta^{2}} \\ 0 & \frac{1}{8 \Delta^{3}} & \frac{-12}{8 \Delta^{3}} & \frac{57}{8 \Delta^{3}} & \frac{-136}{8 \Delta^{3}} & \frac{777}{8 \Delta^{3}} & \frac{-103}{8 \Delta^{3}} & \frac{27}{8 \Delta^{3}}\end{array}\right]\left[\begin{array}{c}v_{n-7} \\ v_{n-6} \\ v_{n-5} \\ v_{n-4} \\ v_{n-3} \\ v_{n-2} \\ v_{m-1} \\ v_{n}\end{array}\right]$
$\hat{\mathbf{v}}=\mathbf{D}_{22} \mathbf{v}$

Another approach to obtain the discretization matrix is to set the degree of freedom with equations obtained from low order discretization (8), thus $\mathbf{D}_{\mathbf{2 1}}$ is obtained for a Gear-2 approach also.

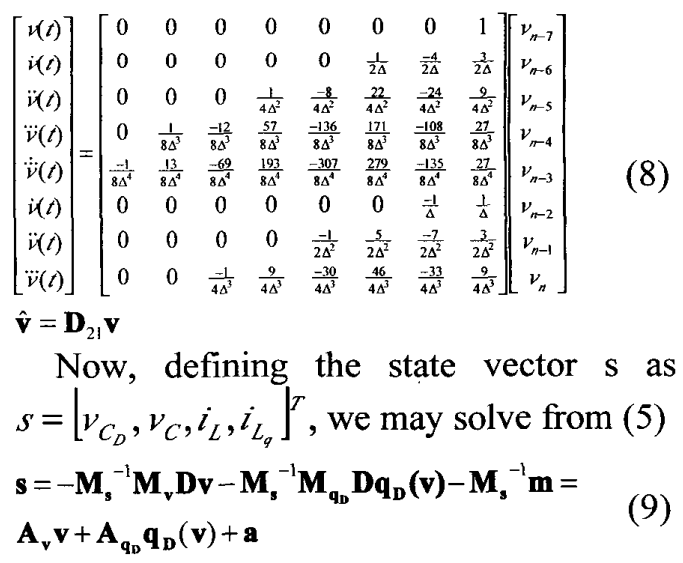

$$
\begin{aligned}
& {\left[\begin{array}{cccc}
1 & 0 & 0 & 0 \\
\frac{-1}{R_{S}} & 0 & -1 & 1 \\
\frac{-1}{L q}-\frac{1}{L} & \frac{1}{L} & \frac{R}{L} & 0 \\
\frac{R}{L^{2}} & \frac{-R}{L^{2}} & \frac{1}{L C}-\frac{R^{2}}{L^{2}} & 0
\end{array}\right]\left[\begin{array}{c}
v_{C_{D}} \\
v_{C} \\
i_{L} \\
i_{L q}
\end{array}\right]+\left[\begin{array}{cccc}
-1 & 0 & 0 & 0 \\
0 & 0 & 0 & 0 \\
0 & \frac{-1}{R_{S}} & 0 & 0 \\
0 & \frac{-1}{L q}-\frac{1}{L} & \frac{-1}{R_{S}} & 0
\end{array}\right]\left[\begin{array}{c}
v(t) \\
\dot{v}(t) \\
\ddot{v}(t) \\
\ddot{v}(t)
\end{array}\right]+\left[\begin{array}{cccc}
0 & 0 & 0 & 0 \\
0 & -1 & 0 & 0 \\
0 & 0 & -1 & 0 \\
0 & 0 & 0 & -1
\end{array}\right]\left[\begin{array}{l}
q_{D}(v) \\
\dot{q}_{D}(v) \\
\ddot{q}_{D}(v) \\
\ddot{q}_{D}(v)
\end{array}\right]+\left[\begin{array}{c}
0 \\
\frac{-V_{C C}}{L q} \\
0 \\
0
\end{array}\right]=0} \\
& \mathbf{M}_{\mathbf{s} O N} \mathbf{s}+\mathbf{M}_{\mathbf{v} O N} \hat{\mathbf{v}}+\mathbf{M}_{\mathbf{q}_{\mathbf{p}} O S} \hat{\mathbf{q}}_{\mathbf{p}}(v)+\mathbf{m}_{O N}=0
\end{aligned}
$$


From this equation we may compute the state vector at the end of one switching period, using the last samples of $v$ a $q_{D}$. If the state vector is continuous when switching occurs,

$\mathbf{s}_{\text {ON }}=\mathbf{s}_{\text {OFF }}$

Or equivalently,

$$
\begin{aligned}
& \mathbf{A}_{\mathbf{v}_{\text {ON }}} \mathbf{v}_{\text {ON }}^{\prime}+\mathbf{A}_{\mathbf{q}_{\text {DON }}} \mathbf{q}_{\text {DON }_{\text {ON }}^{\prime}}^{\prime}+\mathbf{a}_{\text {ON }}= \\
& \mathbf{A}_{\mathbf{v}_{\text {OFP }}} \mathbf{v}_{\text {OFF }}+\mathbf{A}_{\mathbf{q}_{\text {DOFF }}} \mathbf{q}_{\mathbf{D}_{\text {OFF }}}+\mathbf{a}_{\text {OFF }}
\end{aligned}
$$

Where a vector of "initial samples" $\mathbf{v}$ ' is obtained from the last samples of the previous topology.

Transient analysis could be carried out by repeatedly solving (2) and using (11) at each switching.

We may now introduce the periodicity assumption, i.e. $v_{N+1}=v_{1}, v_{N+2}=v_{2}, \ldots$

This leads to the nonlinear algebraic system of $N=N_{O N}+N_{O N}+N_{O F F}+N_{O F F}$ equations described by (12) where the vector of unknowns contains the initial samples $\mathbf{v}$ 'on and v'ofr along with von and voFf. The $N_{O N}$ equations describe the topology of circuit during the ON interval. The $N_{\text {OFF }}$ equations describe the topology during the OFF interval. In this way we have defined the steady-state circuit behaviour in a single matrix. Note that the system is extended with $N^{\prime}{ }_{O N}$ and $N^{\prime} O F F$ equations from (5) due to the added unknowns: the initial samples of $v(t)$ at every switching instant.
The efficient resolution of the nonlinear system of equations is obtained using global convergent methods based on modifications of Newton's method [2].

\section{RESULTS}

This method has been applied to the resolution of the circuit of figure 1 based on [3]. The circuit element values are: $V_{D D}=2 \mathrm{~V}, \quad L_{q}=200 \mathrm{nH}, \quad R_{S}=0.25 \Omega$, $L=2.71 \mathrm{nH}, \quad C=14 \mathrm{pF}, \quad R=1.85 \Omega ., \quad n=3$, $C_{j 0}=40.3 \mathrm{pF}$ and $V_{b i}=1 \mathrm{~V}$.

To avoid convergence problems solving the resulting system of nonlinear equations, the original expression of the nonlinear capacitor

$q_{D}(v)=(n+1) C_{j 0} v_{b i}\left(1+\frac{v}{v_{b i}}\right)^{1 / n+1}$ should be modified [4]. It has shown to be convenient to linearize the behaviour for high levels of $v$. The singularity has also been removed with a linear extension of $q_{D}(v)$ for low levels of $\nu$.

Thus the non-linear charge of capacitor $C_{D}$ is defined in (7).

$$
\begin{cases}q_{D}(v)=m_{d} v+b_{d} & \\ q_{D}(v)=(n+1) C_{j 0} v_{b i}\left(1+\frac{v}{v_{b i}}\right)^{v_{a+1}} & l_{d} v_{b i}>v(t) \\ l_{D} v_{b i}<v(t)<l_{u} \nu+l_{u} v_{b i} & l_{u} v_{b i}<v(t)\end{cases}
$$

We consider $l u=40$ and $l d=-0.85$ the points of linealizing by tangent straight line. The parameters of these lines are defined in (14).

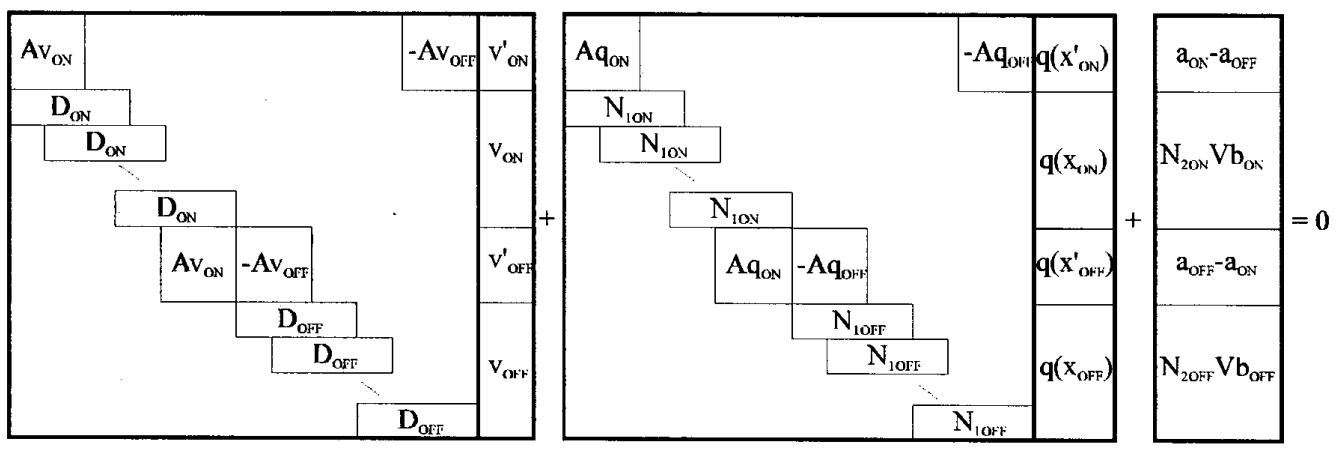




$$
\begin{aligned}
& \left\{\begin{array}{l}
m_{d}=C_{j 0}\left(1+l_{d}\right)^{-n / n+1} \\
b_{d}=(n+1) C_{j 0} v_{b i}\left(1+l_{d d}\right)^{1 / n+1}-m_{d} l_{i} v_{b i}
\end{array}\right. \\
& \left\{\begin{array}{l}
m_{u}=C_{j 0}\left(1+l_{u}\right)^{-n / n+1} \\
b_{u}=(n+1) C_{j 0} v_{b i}\left(1+l_{u}\right)^{1 / n+1}-m_{u} l_{u} v_{b i}
\end{array}\right.
\end{aligned}
$$

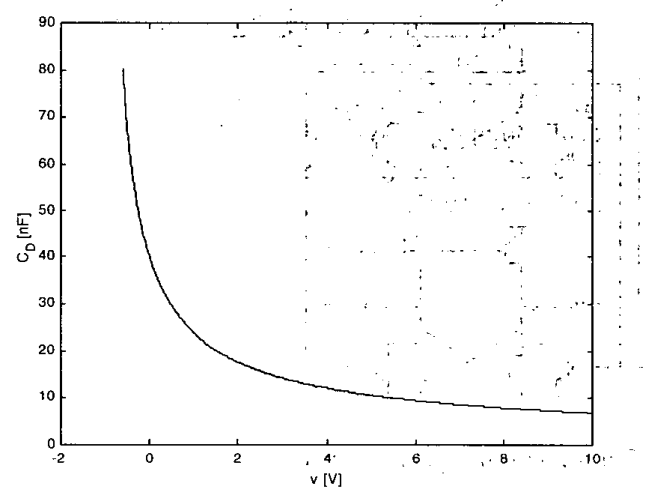

Figure 3. Waveform of $C_{D}(v)$.

The result of $v(t)$ with a discretization of Gear 2 with 20 samples is showed in figure 2. This result agrees with Pspice with 60 samples.

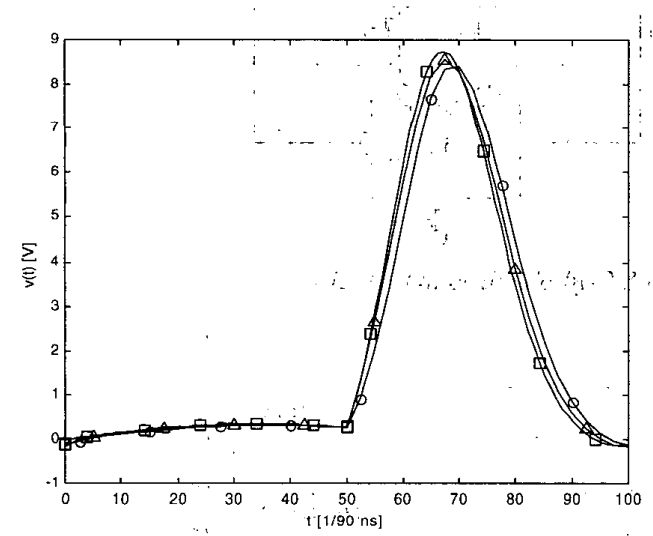

Figure 2. Waveform of $v(t)$. Pspicel (). Gear-2 with $\boldsymbol{D}_{22}(O)$ : : Gear-2 with $\boldsymbol{D}_{21}(\Delta)$.

The result of $v(t)$ with more samples is very accurate and the error isn't appreciate at the chart.

\section{CONCLUSIONS}

A new method to determine the steady state response of nonlinear switched circuits has been presented. The method is based on the time-domain discretization of the equations that describe the circuit [1], transforming: the initial problem, the ' solution of a nonlinear difference differential. system of equations, into the solution of a nonlinear algebraic system. of equations, where the only unknowns to be determined are the samples of the control variables. In "In switched circuits, it is necessary .. to preserve state variable continuity at each switching instant. So, an exact analytic relation between the unknowns and the state variables of the circuit has been described in detail.

To validate the method, it has been applied to the determination of the steady state response of the class-E amplifier, an example of the kind of circuits to which this paper'refers. The results coincide with those obtained using : integration techniques, without having to compute the response until the transient dies out.

\section{IV.REFERENCES}

[1] . - J. Bonet-Dalmau and P. Palà-Schönwälder, "A Discrete-Time Approach to the Steady-State and Stability Analysis of Distributed Nonlinear Autonomous -Circuits" IEEE Trans. Circuits Systems. I, vol 47, pp.231-235, 2000.

[2] J. E. Dennis and R. B. Schnabel, Numerical Methods for Unconstrained Optimization and Nonlinear Equations, Englewood Cliffs, NJ, Prentice Hall (1983).

[3] P. Alinikula, K. Choi and S. I. Long, "Design of Class E Power Amplifier with Nonlinear Parasitic Output Capacitance"; IEEE Transactions on Circuits and Systems, 46, 2, 114:119 (1999).

[4] P. J. C. Rodrigues, Computer-Aided Analysis of Nonlinear. Microwaive Circuits, Artech House (1998). 